\title{
In vivo cartilage-formation potency of somatic stem cells is associated with responsiveness to TGF $\beta$ stimulation in vitro
}

Ryota Chijimatsu ( $\square$ rchijimatsu@g.ecc.u-tokyo.ac.jp)

The University of Tokyo: Tokyo Daigaku https://orcid.org/0000-0002-3125-4818

\section{Satoshi Miwa}

The University of Tokyo: Tokyo Daigaku

Gensuke Okamura

Osaka Rosai Hospital: Osaka Rosai Byoin

Junya Miyahara

The University of Tokyo: Tokyo Daigaku

Naohiro Tachibana

The University of Tokyo: Tokyo Daigaku

Hisatoshi Ishikura

The University of Tokyo: Tokyo Daigaku

Junya Higuchi

The University of Tokyo: Tokyo Daigaku

Yuji Maenohara

The University of Tokyo: Tokyo Daigaku

Shinsaku Tsuji

Avenue Cell Clinic

Shin Sameshima

The University of Tokyo: Tokyo Daigaku

Kentaro Takagi

The University of Tokyo: Tokyo Daigaku

Keiu Nakazato

The University of Tokyo: Tokyo Daigaku

Kohei Kawaguchi

The University of Tokyo: Tokyo Daigaku

Ryota Yamagami

The University of Tokyo: Tokyo Daigaku

Hiroshi Inui

The University of Tokyo: Tokyo Daigaku

Shuji Taketomi 
The University of Tokyo: Tokyo Daigaku

\section{Sakae Tanaka}

The University of Tokyo: Tokyo Daigaku

\section{Taku Saito}

The University of Tokyo: Tokyo Daigaku

\section{Research Article}

Keywords: Chondrogenesis, Stem cell transplantation, transforming growth factor $\beta$ (TGF-b), adipose stem cells, Somatic stem cells

Posted Date: April 5th, 2021

DOl: https://doi.org/10.21203/rs.3.rs-385690/v1

License: (c) (i) This work is licensed under a Creative Commons Attribution 4.0 International License. Read Full License

Version of Record: A version of this preprint was published at Stem Cell Research \& Therapy on July 15th, 2021. See the published version at https://doi.org/10.1186/s13287-021-02485-5. 


\section{Abstract}

Background: Somatic stem cell transplantation has been performed for cartilage injury, but the reparative mechanisms are still conflicting. The chondrogenic potential of stem cells are thought as promising features for cartilage therapy, however the correlation between their potential for chondrogenesis in vitro and in vivo remains undefined. The purpose of this study was to investigate the intrinsic chondrogenic condition depends on cell types and explore an indicator to select useful stem cells for cartilage regeneration.

Methods: The chondrogenic potential of two different stem cell types derived from adipose tissue (ASCs) and synovium (SSCs) of mice and humans was assessed using bone morphogenic protein-2 (BMP2) and transforming growth factor- $\beta 1$ (TGF $\beta 1$ ). Their reparative potential was also validated through transplantation into a mouse osteochondral defect model.

Results: All cell types showed apparent chondrogenesis under the combination of BMP2 and TGF $\beta 1$ in vitro, as assessed by the formation of proteoglycan- and type 2 collagen (COL2)-rich tissues. However, our results vastly differed with those observed following single stimulation among species and cell types; apparent chondrogenesis of mouse ASCs, mouse SSCs, and human SSCs was observed with supplementation of BMP2, either BMP2 and TGF $\beta 1$, and BMP2, respectively. Human ASCs showed no chondrogenesis following single stimulation. Among human SSCs, two of six donors formed partially COL2-positive tissues in response to TGF $\beta 1$. However, unlike mouse cells, human cells showed fibrous components (COL1/3) with all treatments. Mouse SSCs formed hyaline-like cartilage (proteoglycan+/COL2+/COL1-/COL3-) in the transplanted site in vivo, but other cell types mainly formed COL1/3-positive fibrous tissues. Remarkably, only donors that showed chondrogenic response to TGF $\beta 1$ in vitro formed partially COL2-positive tissues in vivo. However, the area was mainly composed of COL $1 / 3$, indicating fibrous cartilage-like tissues.

Conclusion: The chondrogenic response to TGF $\beta 1$ may represent the fate of stem cells when locally transplanted into cartilage defects.

\section{Introduction}

It is widely accepted that local injury of articular cartilage associated with joint trauma does not regenerate spontaneously. Transplantation of mesenchymal stem cells (MSCs) derived from bone marrow, synovium, and adipose tissue has shown to be a promising strategy for cartilage regeneration. Several clinical trials using MSCs have been completed or are ongoing [1, 2], although the actual outcome of transplantation of MSCs remain unclear.

There are conflicting reports on the reparative mechanisms of local transplantation of MSCs. Koga et al. reported that transplantation of bone marrow MSCs (BMSCs) or synovial MSCs (SMSCs) repaired cartilage defects with differentiation of transplanted cells to chondrocytes in an in vivo environment in a rabbit osteochondral defect model [3]. After transplantation, the cells were engrafted and functioned as 
chondrocytes/cartilage over six months [4]. On the other hand, Nakamura et al. found in a porcine study that the transplanted SMSCs repaired the cartilage defect without differentiation to chondrocytes; moreover, the transplanted SMSCs disappeared within a month [5]. Recently, many studies have highlighted that the signaling effects of transplanted stem cells lead to endogenous repair by host stem cells through the secretion of growth factors, cytokines, microRNAs, extracellular vesicles, and/or cell-cell contact $[6,7]$. Thus, distinct mechanisms may be involved in transplantation therapy.

Several studies have suggested a relationship between in vitro chondrogenic potential and in vivo cartilage regeneration $[3,8,9]$. Among MSCs, SMSCs are reported to provide superior chondrogenic potential in humans $[10,11]$, rodents $[12,13]$, rabbit [3], and large animals $[5,14]$. Based on those findings, clinical trials of transplantation of SMSCs for articular cartilage defects have been recently reported [15, 16]. However, Dickhut et al. showed that transforming growth factor- $\beta 3$ (TGF $\beta 3$ ) completely induced chondrogenesis from BMSCs, which was evident with the formation of tissue rich in type 2 collagen (COL2) and proteoglycan in vitro, whereas the stimulation with TGF $\beta$ s alone was insufficient for SMSCs and adipose MSCs (AMSCs). The formed tissues contained a low amount of glycosaminoglycans and COL2 $[17,18]$. Previous studies have shown that supplementation with bone morphogenic proteins (BMPs) is necessary for induction of chondrogenesis from SMSCs and AMSCs [17, 19-21]. Moreover, other supplements such as serum and glucocorticoids also affect the chondrogenesis [19, 22-26]. Thus, conditions for adequate chondrogenesis are intrinsically different among MSCs, implying that chondrogenic potential is probably miscalculated depending on assay conditions.

For further understanding the mechanisms underlying the action of stem cells transplantation for cartilage repair therapy, we aimed to evaluate the in vitro and in vivo chondrogenic potential of two different somatic stem cell types derived from synovium and adipose tissue in mice and humans, respectively.

\section{Materials And Methods}

\section{Isolation and culture of somatic stem cells}

Mouse somatic stem cells were isolated from 8-10-week-old C57BL/6 or CAG-EGFP C57BL/6. Mouse synovial stem cells (mSSCs) were established from knee infrapatellar fat pad as previously reported with slight modifications $[13,27]$. In brief, synovium containing infrapatellar fat pad was surgically dissected and incubated in $10 \%$ fetal bovine serum (FBS; Sigma-Aldrich, MO, USA)-DMEM (Nacalai Tesque (Nacalai), Kyoto, Japan) containing $500 \mathrm{U} / \mathrm{mL}$ collagenase type 1 (Worthington, $\mathrm{NJ}, \mathrm{USA}$ ) at $37^{\circ} \mathrm{C}$ with gentle rotation. After $1 \mathrm{~h}$, the digested tissues were passed through a $70-\mu \mathrm{m}$ strainer and washed twice. The isolated cells were cultured with growth media (10\% FBS-DMEM supplemented with $1 \mathrm{ng} / \mathrm{mL} b F G$ (FUJIFILM Wako Pure Chemical Corporation (Wako), Osaka, Japan) at $37^{\circ} \mathrm{C}$ in a humidified atmosphere with $5 \% \mathrm{CO}_{2}$ and $3 \% \mathrm{O}_{2}$. Mouse adipose stem cells (mASCs) were obtained from the stromal vascular fraction of the inguinal fat tissue by collagenase digestion for $30 \mathrm{~min}$. Only dissociated cells were collected using a $70-\mu \mathrm{m}$ strainer and cultured with growth media at $37^{\circ} \mathrm{C}$ in a humidified atmosphere with 
$5 \% \mathrm{CO}_{2}$ and $3 \% \mathrm{O}_{2}$. Cultured cells were passaged with $0.25 \%$ trypsin/ethylenediaminetetraacetic acid (EDTA) at $80 \%$ confluency and replated at a density of $5,000 \mathrm{cells} / \mathrm{cm}_{2}$. The medium was changed three times per week. These cells have been already characterized in our previous report [27]. The cells were used for further experiments after 12-14 days of culture, as mouse somatic stem cells are sensitive to senescence $[27,28]$. We prepared over three batches obtained from several mice with mixed gender at different timings.

Human subcutaneous adipose tissue and synovium were obtained from osteoarthritis (OA) patients $(\mathrm{N}=6)$ during total knee arthroplasty (TKA) in accordance with a protocol approved by the institutional ethics committee. Written informed consent was obtained from all patients. The age, gender, and Kellgren-Lawrence grade of the patients are listed in Additional file 1. Adipose tissues were resected from the incision site at the knee, and synovium was resected from the suprapatellar pouch. Human ASCs (hASCs) were isolated as mentioned above and human SSCs (hSSCs) were isolated as previously established [18]. Human cells were cultured with growth media at $37^{\circ} \mathrm{C}$ in a humidified atmosphere with $5 \% \mathrm{CO}_{2}$. Those cells were passaged by treatment with $0.25 \%$ trypsin/EDTA at $80 \%$ confluency (approximately once per week) and replated at a density of $5,000 \mathrm{cells} / \mathrm{cm}_{2}$. The media was changed twice per week. Human cells at passage 3 were used for further experiments.

\section{Chondrogenic induction}

To obtain cell pellets, $2 \times 10^{5}$ cells were centrifuged in polypropylene tube and cultured in growth medium. The next day, the medium was exchanged to chondrogenic medium (DMEM, $1 \%$ ITS+Premix (Corning, NY, USA), $50 \mu \mathrm{g} / \mathrm{mL}$ L-ascorbic acid 2-phosphate (Sigma-Aldrich), $40 \mu \mathrm{g} / \mathrm{mL}$ L-proline (SigmaAldrich)) supplemented with $100 \mathrm{ng} / \mathrm{mL}$ BMP2 (Medtronic, Dublin, Ireland), $10 \mathrm{ng} / \mathrm{mL}$ TGF $\beta 1$ (ORIENTAL YEAST, Tokyo, Japan). Dexamethasone (Sigma-Aldrich) was used with TGF $\beta 1$ at concentration of $10 \mathrm{nM}$ [19]. The cell pellets were maintained with $0.5 \mathrm{~mL}$ medium at $37^{\circ} \mathrm{C}$ in a humidified atmosphere with $5 \%$ $\mathrm{CO}_{2}$. The medium was replaced twice per week.

\section{Animal experiment}

Twelve-week-old male mice were anesthetized by an intraperitoneal injection of a mixture of $0.3 \mathrm{mg} / \mathrm{kg}$ of medetomidine, $4.0 \mathrm{mg} / \mathrm{kg}$ of midazolam, and $5.0 \mathrm{mg} / \mathrm{kg}$ of butorphanol. For both knees, the femoral trochlear grooves were exposed via a medial parapatellar incision with laterally patellar dislocation. An osteochondral defect (diameter, $0.5 \mathrm{~mm}$; depth, approximately $0.5 \mathrm{~mm}$ ) was created in both knees by manual drilling. A pellet of $5 \times 10^{4}$ cells prepared by overnight culture with growth media were transplanted into the defects, and then patellar dislocation was reduced. The joint capsule and the skin were sutured as separate layers. After surgery, mice were allowed to be active without any fixation device or immobilization. C57BL/ 6 and CAG-EGFP C57BL/ 6 mice were used for allograft study and C.B-17 SCID mice were used for xenograft study.

\section{Micro-computed tomography}


Whole knee joints were scanned using micro-computed tomography $(\mu C T$; inspeXio SMX-100CT system (Shimadzu, Kyoto, Japan)) at a resolution of $12 \mu \mathrm{m}$ per voxel using the following consistent parameters: $75 \mathrm{kV}$ and $140 \mathrm{~mA}$. Three-dimensional images of bone were analyzed using the TRI/3D-BON software (RATOC System Engineering, Tokyo, Japan). Bone volume (BV) and bone mineral density (BMD) at the area of osteochondral defect trimmed into a cylinder (diameter, $0.5 \mathrm{~mm}$; depth, $0.4 \mathrm{~mm}$ ) were calculated as described previously [29].

\section{Histology and histochemistry}

The samples were fixed in $10 \%$ neutral buffered formalin and embedded in paraffin wax, which was followed by serial dehydration using ethanol and clearance using xylene. For bone tissues, samples were decalcified with 10\% EDTA (pH 7.4) after formalin fixation and delipidation using ethanol. Sections cut into $4 \mu \mathrm{m}$ thickness were used for Safranin-O/fast green/hematoxylin staining (Saf-0), Alcian blue ( $\mathrm{pH}$ 1.0) staining (AB), Tartrate-resistant acid phosphatase (TRAP) staining, and immunohistochemistry. The information regarding the antibodies and reaction conditions is listed in Additional file 2 After reaction with HPR or AP conjugated antibodies, positive signal color was developed with Histofine Simple stain DAB (NICHIREI BIOSCIENCES, Tokyo, Japan), Vina Green Chromogen Kit (BIOCARE MEDICAL, CA, USA), or ImmPACT Vector Red AP Substrate Kit (VECTOR LABORATORIES, CA, USA). Positively stained areas were measured using Image J (National Institutes of Health, MD, USA), ImageScope (Leica Microsystems, Wetzlar, Germany), and BZX-700 (Keyence, Osaka, Japan).

\section{mRNA expression analysis}

Cell pellets were initially homogenized with zirconia beads in TRI Reagent (Cosmo Bio, Tokyo, Japan). Total RNA was then extracted using Direct-zol RNA Kit (Zymo Research, CA, USA) according to the manufacturer's protocol. Total RNA was reverse transcribed to cDNA using ReverTra Ace qPCR RT Master Mix (TOYOBO, Osaka, Japan). Quantitative reverse transcription polymerase chain reaction was performed using THUNDERBIRD SYBR qPCR Mix (TOYOBO) and Thermal Cycler Dice Real Time System III (TaKaRa Bio, Shiga, Japan). The information about primers is listed in Additional file 3. The expression levels normalized to the levels of glyceraldehyde 3-phosphate dehydrogenase (GAPDH) were measured using absolute standard curve method [30] or delta CT method [31].

\section{Transcriptome Analysis}

Total RNA extracted from human cells was used for transcriptome sequencing. The sequence libraries was prepared using a NEBNext Ultra II RNA Library Prep Kit for Illumina (New England Biolabs, MA, USA) according to the manufacture's protocol. Sequencing was performed using an Illumina HiSeq 4000 System with $2 \times 150$ bp paired-end reads (Veritas Genetics, MA, USA). The raw sequence data were filtered to remove adaptor sequences, low-quality reads, sequences with a high content of $\mathrm{N}$, and reads $<50 \mathrm{bp}$ length by using Trimmomatic (ver.0.39). The filtered data were aligned against the human reference genome (GRCh38.p13) using STAR (ver.2.7.3a). The gene expression counts and transcripts per million value (TPM) were calculated by RSEM (ver.1.3.3). Principal component analysis and hierarchical 
clustering was conducted by iDEP.91 (http://bioinformatics.sdstate.edu/idep/). For enrichment analysis in specific pathways, genes are collected from several gene sets related to TGF $\beta$ receptor signaling (M2642), Proteoglycan (M15611, M12097, M13795, and M13500), and Chondrogenesis/Cartilage development (M10512, M14448, M11632, M34061, M15986, M10632, and M13025) from MSigDB v6.0. Heatmaps were created in R packages based on Log2(TPM+1) or Z score of TPM values.

\section{Western blotting}

Cells were lysed with T-PER (Thermo Fisher Scientific (Thermo), MA, USA) containing protease and phosphatase inhibitors (Nacalai). Following mixing with 4x LDS sample buffer (Thermo) and DTT (Nacalai), the samples were boiled at $70^{\circ} \mathrm{C}$ for $10 \mathrm{~min} ; 10 \mu \mathrm{g}$ protein was applied to each lane of $4-12 \%$ Bolt Bis-Tris Plus precast polyacrylamide gel (Thermo) and separated by electrophoresis. For cell pellets, three replicates were pooled, and one-fifth volume of lysate was used for electrophoresis. Subsequently, the gels were transferred onto a PVDF membrane (Wako) using a Mini Blot Module (Thermo). After blocking with Blocking One (Nacalai) for $30 \mathrm{~min}$, the membranes were probed with the following antibodies overnight at $4^{\circ} \mathrm{C}$ or $1 \mathrm{~h}$ at room temperature: anti-phospho-Smad1/5 (\#9516, CST, MA, USA), anti-Smad1 (\#9743, CST), anti-phospho-Smad2 (\#3108, CST), and anti-Smad2/3 (\#8685, CST), antiSOX9 (\#82630, CST), HRP-conjugated-anti-GAPDH (\#HRP-60004, Proteintech, IL, USA), HRP-conjugatedanti-rabbit IgG (\#7074, CST). Then, immunoreaction was visualized with ChemiLumi One Super (Nacalai) and iBright 1000 (Thermo). The band signals were measured using iBright Analysis Software (Thermo).

\section{Results}

\section{TGF 1 induced chondrogenesis from mSSCs but not mASCs in vitro}

Previous reports indicate that optimal chondrogenic conditions differ among cell types [17, 32, 33]. To evaluate the actual chondrogenic capacity, in vitro pellet culture under stimulation with BMP2, TGF $\beta 1$, or their combination $(B+T)$ was performed. As a reference, neither mASCs nor mSSCs differentiated into chondrocyte in the basal chondrogenic medium (see Additional file 4).

When mASC pellets were cultured under those three conditions, chondrogenic differentiation ascertained by semi-translucent tissue formation composed of proteoglycan and COL2 was recognized with supplementation of BMP2 (Fig. 1A, 1B). However, fibrotic matrices composed of type 1 and 3 collagen were observed in the chondrogenic tissues, indicating fibrocartilage development. Although there was no evidence for chondrogenesis in TGF $\beta 1$ treated mASCs even at the mRNA levels (see Additional file 5), TGF $\beta 1$ facilitated BMP2 induced chondrogenesis with reduction of COL1/3 component. In contrast, mSSCs underwent chondrogenesis under all conditions with the formation of a semi-translucent tissue with SOX9-positive chondrocytes embedded in the homogenous extracellular matrices enriched in proteoglycan, type 2 collagen, and low amount of COL1/3 (Fig. 1C, 1D). These observations show that mSSCs could form hyaline-like cartilaginous tissue. COL10, a hypertrophic marker, was observed in the 
area surrounding the hypertrophic cell lacuna only with BMP2 supplementation. The same trends were observed and validated at gene levels (see Additional file 5). Taken together, mASCs and mSSCs showed the potential for chondrogenesis but the suitable conditions were intrinsically different among the various cell types in vitro.

\section{mSSCs, but not mASCs, formed cartilaginous tissue in the osteochondral defect in vivo}

The reparative capacities of mASCs and mSSCs in the osteochondral defect were evaluated by transplantation of the cell pellet into osteochondral defects. The osteochondral defect with no transplantation showed spontaneous repair of the subchondral bone, but the articular cartilage region did not regenerate, and was covered with fibrous tissue $(N=11 / 14)$ or exposed bone $(N=3 / 14)$ (Fig. $2 A)$. When mASCs were transplanted, the defects were filled with fibrous tissue with no regeneration of articular cartilage (Fig. 2B). Although, COL1 is a major fibrous tissue component, its detection was complicated for the assessment of fibrous tissue because the surrounding bone was also positive for COL1. Therefore, COL3, which is another fibrous component, was adapted in this study and strikingly signified the fibrotic area. GFP detection demonstrated that transplanted cells remained within the transplanted site without differentiation toward chondrocytes or osteoblasts, resulting in the impeded spontaneous repair of subchondral bone. In some cases, Saf- $\mathrm{O}^{+} / \mathrm{COL} 2^{+}$area was partially observed $(\mathrm{N}=$ $4 / 25)$; however, they were fibrous $(N=3 / 25)$ or ectopic site that protruded from the articular cartilage region $(N=1 / 25)$ (see Additional file 6).

On the other hand, when mSSCs were transplanted into the chondral defect, cartilaginous tissue rich in proteoglycans and COL2 but low in COL1/3 $(N=15 / 20)$ was newly generated (Fig. 2C). Interestingly, in all unrepaired cases $(N=5 / 20)$, defect sites were located in the distal femur groove and the host synovium tissue was infiltrated from enthesis of crucial ligaments to the defect site (see Additional file 7). GFP mice-derived mSSCs were detected at neo cartilage tissue, indicating that mSSCs differentiated to chondrocytes and formed cartilage tissue in vivo (Fig. 2D). Notably, GFP-negative cells were also detected within the neo-cartilage tissue, particularly in the articular surface area and the boundary of bone marrow. To investigate the contribution of host cells, wild-type (WT)-derived mSSCs were transplanted into GFP mice. As a result, approximately $20 \%$ host cells were also incorporated for cartilage regeneration with differentiation toward chondrocytes (GFP-positive cell rate: $83.1 \pm 6.6 \%(\mathrm{~N}=5)$ in GFP cell transplantation to WT mice and $20.8 \pm 11.4 \%(\mathrm{~N}=4)$ in WT cell transplantation to GFP mice). At 6 weeks, remodeling of the neo-cartilaginous tissue was histologically observed in the deep zone (Fig. 2E). COL10 was observed from the middle to the deeper area of the neo-cartilage, which expanded at 6 weeks. The $\mu \mathrm{CT}$ analysis revealed that ossified volume of neo-tissues increased at 6 weeks but the mineral density did not matured like bone, suggesting the proceeding cartilage ossification (Fig. 2F). These findings indicate that mSSCs repaired the osteochondral defect via endochondral development, and that accompanied with the stimulation of host cells to differentiate toward chondrocytes. 


\section{TGF $\beta$ receptor derived-signaling is necessary for chondrogenesis}

MASCs and mSSCs demonstrated chondrogenic potential in vitro but only mSSCs could differentiate to chondrocytes in vivo. Based on these results, it should be specified that the chondrogenic response to TGF $\beta 1$ was observed only in mSSCs in vitro. Phosphorylated Smad2/3 and Smad1/5/8 are the major downstream of TGF 31 and BMP2 signaling, respectively [34]. In vivo specimens transplanted with mSSCs showed strong pSmad1 and weak pSmad2 activation during chondrogenesis. On the other hand, the fibrous area formed by mASCs contained cells weakly positive for pSmad1 and negative for pSmad2 (see Additional file 8). Therefore, the TGF $\beta$-Smad2/3 pathway is important during in vivo chondrogenesis.

To elucidate the distinct outcome in response to TGF $\beta 1$ among cell types, the expression of their specific receptors was confirmed. Among TGF receptor superfamilies, Alk1 and Alk7 were remarkably higher in mSSCs and mASCs, respectively. However, Alk5 which is a major TGF $\beta$ type 1 receptor, and Tgfbr2 were comparable in mSSCs and mASCs. There was no difference in Alk2, Alk3, Alk4, Alk6, and Bmpr2 (Fig. 3A). Furthermore, the levels of most receptors did not dynamically alter in 2 weeks of chondrogenic culture (see Additional file 9A). Western blotting analysis showed that during short-term stimulation, Smad1/5 and Smad2 were activated in response to BMP2 and TGF $\beta 1$, respectively, in both cell types according to conventional theory (see Additional file 10). Thus, mASCs and mSSCs were comparable in terms of the expression of TGF $\beta$ receptors as well as of receptors mediating Smads phosphorylation.

Conversely, in chondrogenic pellet culture, TGF $\beta 1$ did not affect either Smad1/5 and Smad2 pathway in mASCs, while it temporally activated Smad2 and Smad1/5 in some batches of mSSCs with obscure trends (Figs. 3B, see Additional file 11). Notably, the activation of Smad2 was marked with the presence of BMP2 as well as Smad1/5 in both cell types. This was supported by the fact that SB-431542, an Alk5 inhibitor, strikingly abrogated chondrogenesis induced not only by TGF $\beta 1$ but also by BMP2 (Fig. 3C). Although TGF $\beta$ signal is associated with cell growth and survival, its inhibition led adipogenesis not cell death (see Additional file 12).

LDN-193189, a BMP receptors (Alk2 and Alk3) inhibitor, also weakened TGF $\beta 1$-induced chondrogenesis of mSSCs to a lesser extent than it weakened BMP2-induced chondrogenesis (Fig. $3 \mathrm{C}$ ) as 10-fold higher dose of LDN-193189 was required to inhibit TGF $\beta 1$-induced chondrogenesis (see Additional file 13). Considering the activation of Smad2 during BMP2 stimulation, it is possible that autocrine ligand expression contributed to chondrogenesis. Regarding endogenous chondrogenic growth factors, Bmp2 was expressed higher in mSSCs than mASCs whereas TGF $\beta 1 / 3$ were expressed at comparable levels (Fig. 3D). Bmp4 and Bmp7 were barely expressed in two cell types (data not shown). Tgfb1/3 were constant or marginally upregulated in chondrogenesis, and Bmp2 and Bmp6 were upregulated in BMP2 stimulation toward the end of induction, similar to Col10a1 (see Additional file 9B).

Based on the above in vitro findings, it can be inferred that TGF $\beta$ receptor-mediated signal was indispensable for chondrogenesis. However, the output in response to TGF $\beta$ could not be determined in 
their profiles for receptors and ligands.

\section{Distinct chondrogenic conditions and outcomes between hASCs and hSSCs with variations among donors}

To expand the scope of application of murine experiments in human somatic stem cells, the responses of hASCs and hSSCs to chondrogenic growth factors were evaluated in vitro.

When hASCs were cultured with BMP2 or TGF $\beta 1$ alone, weak staining for Saf-O and aggrecan was recognized in the formed tissues in some cases but COL2 was completely absent (Fig. 4A). Instead, COL1/3 were abundant in their tissues. Their chondrogenic potential could be demonstrated with use of the combination of BMP2 and TGF $\beta 1$, as evident from the development of proteoglycan- and type 2 collagen-enriched translucent tissue. With increased cartilaginous matrices, COL3 was restricted to the peripheral zone; however, COL1 was observed, similar to that in other single treatments. These results were consistently observed in all six donors (Fig. 4B).

In hSSCs, BMP2 + TGFB1 stably induced chondrogenesis in all donors similar to hASCs, whereas a distinct pattern for chondrogenic response to single stimulation was uniquely exhibited among the donors (Fig. 4C, 4D). When hSSCs were cultured with BMP2 alone, different grades of chondrogenesis were observed among donors: partially intense staining for chondrogenic markers was observed in $3 / 6$ donors (D1, D3, and D4) and entire intense staining in 2/6 donors (D2 and D5) within developed tissues. Conversely, TGF $\beta 1$ alone induced proteoglycan synthesis, as observed in Saf-O and immunohistochemistry for aggrecan. Nevertheless, COL2 was recognized only in 2/6 donors (D1 and D3). Correlation analysis supported the inconsistency of the relationship between proteoglycans and COL2 (see Additional file 14A). Of note, all tissues developed from hASCs and hSSCs clearly contained COL 1/3 under all given chondrogenic conditions. COL3 tended to be weakened in the specimen with intense staining for COL2; however, there was no negative correlation between COL1 and COL2 (see Additional file 14B and 14C).

Interestingly, the chondrogenic potential based on the response to BMP2 was not representative of the chondrogenic response to TGF $\beta 1$ (see Additional file 15). Thus, unique chondrogenic responses were observed between cell types and donors, although principal component analysis plot and hierarchical correlation analysis of mRNA-seq data showed low consistency in gene profiles between hASCs and hSSCs (Figs. 5A, 5B, see Additional file 16 and 17). The expression of receptors and ligands of the TGF $\beta$ superfamily was similar among cell types and donors (Fig. 5C). Moreover, no specific enrichment was observed in multiple gene sets related to TGF $\beta$ signaling, proteoglycan, and chondrogenesis/cartilage development (see Additional file 18).

Thus, hASCs and hSSCs required distinct chondrogenic condition beyond those required by mouse stem cells. Moreover, the fibrous components were more abundant in developed tissues in human stem cell cultures, which characteristically resembled fibrocartilage in human stem cells. However, similar to that in 
mouse stem cells, the chondrogenic potential could not be predicted based on receptor/ligand expression.

\section{Only hSSCs reactive to TGF $\beta 1$ in vitro formed fibrous cartilage in vivo}

To investigate the in vivo chondrogenic potential of human stem cells, hASCs and hSSCs were transplanted into the osteochondral defect model using SCID mice without pre-chondrogenic induction and evaluated at 4 weeks.

In case of hASC transplantation, the subchondral bone was partially regenerated, but the surface zone was filled with fibrous tissues composed of $\operatorname{COL} 1 / 3$, and no cartilaginous matrices were detected in all donors (Figs. 6A, see Additional file 19). HSSCs transplantation revealed that defects did not contain bony tissues; instead, newly formed tissue filled out the defect space in all specimens. Among them, the formation of cartilaginous tissue containing proteoglycan, partial COL2, and SOX9 expressing cells was occasionally recognized in $D 1(N=1 / 4)$ and $D 3(N=4 / 4)$, which exhibited a chondrogenic response to TGF $\beta 1$ in vitro (Figs. 6B, see Additional file 20). Notably, as indicated in vitro, the fibrous component, $\operatorname{COL1/3,~was~present~in~the~entire~defect~site.~Immunohistochemistry~for~human~vimentin~showed~that~}$ hASCs, without any specific differentiation, diffusely remained in tissues with granulation rich in hypercellularity and small vessels (Figs. 6A, see Additional file 21A). On the contrary, hSSCs were better engrafted within the osteochondral region even in cells with the lowest in vitro chondrogenic potential (Figs. 6B, see Additional file 21B). In some cases, the defect area in subchondral bone widened and/or spilled out into host area. Moreover, the erosion sometimes reached to the articular cartilage (see Additional file 21, arrow heads). TRAP activity indicated erosion at the border between the neo-tissue and surrounding bone and cartilage, whereas there was lesser erosion in the periphery of the neo-tissue enriched in aggrecan-positive matrices (see Additional file 21).

Collectively, TGF $\beta 1$-responsive hSSCs could form fibrous cartilage in vivo. Moreover, the engraftment rate was intrinsically different among cell types regardless of the chondrogenic potential. Furthermore, the local transplantation of cells with lower potential for in vivo chondrogenesis may lead to other side effects such as erosion of the bone and cartilage.

\section{Discussion}

Our findings showed that the chondrogenic potential of somatic stem cells in vitro, which has been defined in BMP2 supplemented conditions, did not correlate with the reparative capacity for local transplantation therapy of the osteochondral defect in vivo. Moreover, this study is the first to demonstrate that the response to TGF $\beta 1$ in vitro represents the outcome after local transplantation, which may be a potential indicator to predict in vivo chondrogenesis. Although transplantation therapy of human SSCs have been conducted for the purpose of repair articular cartilage [15, 16], it may be more suitable for fibrous cartilage repair such as meniscus [26, 35, 36]. Moreover, the test for chondrogenic response to TGF $\beta$ in vitro will provide a new criteria to select cell source from allogenic cell banks. 
As the major limitation of a small animal study, the subchondral bone defect must be employed for the purpose of studying only the cartilage region. However, our data showed lower reparability of the cartilage even in an osteochondral injury, whereas bone defect region was spontaneously regenerated with membranous ossification. For articular cartilage repair, cartilaginous callus formation is required to remodel the osteochondral region for endochondral development. As SSCs are known to induce host synovial tissues [36], location of the defect site is critical for mSSC transplantation because infiltration of the host synovial tissue was observed when the defect was close to the enthesis of crucial ligaments. Furthermore, transplanted mSSCs detected below the marrow area did not exhibit signs of chondrogenesis, and in case of synovial infiltration, vessel-like shapes were found (see Additional file 7). Thus, location and site-specific signals, including mechanical stress, may be necessary for in vivo chondrogenesis as chondrogenesis was not observed in the knee with patellar dislocation (data not shown) [37, 38].

Although we concluded the importance of chondrogenic response to TGF $\beta 1$, the distinct chondrogenic result of each cell type could not be elucidated in the analysis of TGF $\beta$ superfamily receptors, endogenous ligands, and downstream pSmads. In fact, all cell types used here were considered to react to TGF $\beta$ with unique tissue formation, unlike that found in basal or BMP2 supplemented medium. Other studies have shown the inconsistencies in cellular receptor expression in in vitro chondrogenic activity $[39,40]$. In general, TGF $\beta$ s and BMPs bind their specific receptors; ALK5 is a type I receptor for TGF $\beta$ s; ALK2, ALK3, and ALK6 are type I receptors for BMPs, which activate downstream Smads, Smad2/3 and Smad1/5/8, respectively. However, TGF $\beta$ s are known to activate Smad1/5/8 pathway via binding to ALK1, a predominant BMP type I receptor [34], and BMPs can activate Smad2 pathway via binding to ALK5 [41]. The pathway that will be activated is determined by the abundance of receptors on the cell membrane and their affinity to ligands [42]. For example, TGF $\beta$ signaling in OA chondrocytes switch from the anabolic ALK5-Smad2/3 pathway to catabolic ALK1-Smad1/5/8 pathway due to disproportionate ALK1/ALK5 ratio, which accelerates cartilage destruction [43-46]. Using western blotting, we demonstrated that Smad activation in the order of hours and days were distinctly regulated. It is known that transcriptional targets of Smad2/3 dynamically change in the order of minutes and hours [47]. These results suggest that there are inadequate downstream gene markers to assess the activation of TGF $\beta$ signaling. Moreover, the non-Smad pathways also regulate cellular function and differentiation, including chondrogenesis $[34,48]$. Thus, the entirety of TGF $\beta$ signaling from cue to output in each cell type is difficult to interpret; therefore, the chondrogenic response to TGF $\beta$ cannot be predicted without chondrogenic culture.

BMP signaling is crucial for skeletogenesis, particularly for endochondral development. On the contrary, TGF $\beta$ signaling is crucial for joint development $[49,50]$ and homeostasis of articular cartilage $[43,51,52]$. In fact, TGF $\beta 1$ is constantly supplied to the synovial fluid from cartilage and synovium $[53,54]$. Therefore, tests for response to TGF $\beta$ can reasonably predict cell fate after transplantation into joints. BMSCs undergo chondrogenesis with TGF $\beta$ treatment alone $[17,55]$, and are responsible for cartilage regeneration in focal cartilage defect using the bone marrow stimulation method [56]. We previously showed the in vivo chondrogenic potential of hBMSCs in a rat osteochondral model [22]. To further verify 
our proposal, we should have included hBMSCs in our analysis; however, as one of the limitations, it was difficult to prepare a sufficient number of hBMSCs from bone marrow effusion after osteotomy in TKA operation. Besides, we used subcutaneous fat from the knee area, which may have different characteristics from abdominal fat $[57,58]$. Recently, medical waste-derived stem cells such as adipose tissue, synovium, umbilical cord, and dental pulp have been highlighted for stem cell therapy because of low additional invasion for tissue collection and their superior proliferative capacity compared with BMSCs $[11,59,60]$. Our data are limited and may not be applicable to other cell types; therefore, further study is required using other sources and cells purified from specific populations.

Unlike hASCs, there are various types of hSSCs among donors. This is because synovial tissue contains various components such as synovial lining, connective tissue, blood vessels, and adipose tissue, which are altered under pathologic conditions [61,62]. Mochizuki et al. showed that adipogenic changes in human synovium caused low chondrogenic potential of their derivates [63], and Mizuno et al. demonstrated that each derivate of the synovial surface, stroma, and perivascular behaves as synovial fibroblasts in vitro with different chondrogenic potential under the combination of TGF 33 and BMP2; among them, the perivascular-derived hSSCs exhibit the highest chondrogenic potential [64]. Furthermore, Sicasubramaniyan et al. showed that primary $\mathrm{CD} 73^{+} / \mathrm{CD} 90^{-}$synovial cells showed a chondrogenic response to TGF $\beta 1$, but $\mathrm{CD} 73^{+} / \mathrm{CD} 90^{+}$synovial cells required additional stimulation with BMP2 to undergo chondrogenesis [39]. The study also pointed a discrepancy in the expression of aggrecan and COL2 in chondrogenic culture of hSSCs, as shown herein as well as in our previous study [18]. Thus, conventional cell isolation from synovium without any selection yields low reproducibility, whereas our results provide a new criterion for selecting a useful cell source, even from OA synovium.

It is known that synovial fibroblasts (SFs) contribute to cartilage and bone erosion in rheumatoid arthritis (RA) [65]. Given that cultured SFs have traditionally been studied in arthritis using the same isolation procedure used in therapeutic study, their erosive potential of hSSCs should be considered. Recent singlecell transcriptome analysis showed that inflammatory fibroblasts expressing TNFa, IL6, and IL1B as well as immune cells were found in OA synovium. Moreover, they also expressed several proteinases such as ADAMTSs and MMPs [66]. Tsuchiya et al. showed that the responses of OA-SFs to the stimulation of inflammatory cytokines were shared in RA-SFs, indicating the common potential for proinflammation in expanded cells derived from OA, RA [67], and probably healthy synovium. Furthermore, other groups demonstrated that hSSCs either from healthy subjects or OA patients could induce osteoclastogenesis from PBMCs in vitro [68]. These results support our data regarding the TRAP activity at the border of fibrous neo-tissues. Altogether, cultured synovial cells have two aspects as stem cells and diseasemodifying fibroblasts. Regarding the purpose of regenerative therapy, further study will be needed to avoid the negative effects of hSSCs. These findings have not been reported in hASCs; however, the erosion of host tissue was also observed in cell transplantation. Our data indicate that local transplantation for chondral injuries using cells with low chondrogenic potential in vivo should be carefully considered. 
There are several limitations in this study. Firstly, we did not verify the surface antigens and potential of cells for osteogenesis and adipogenesis, which are often used in MSC studies; however these have been confirmed in our previous studies $[27,69]$ or elsewhere with comparable culture methods $[17,60]$. In fact, the criteria and terminology for MSCs have recently been reconsidered [70]. Therefore, we referred to somatic stem cells using the name of the source tissue in this study. With respect to repair in bone diseases, evaluation of the osteogenic capacity in vitro may be helpful; however, in vivo osteogenesis was not recognized in the cells studied herein. Secondly, we suggested that endogenous expression of TGF $\beta$ superfamilies may contribute during chondrogenesis, but it was only at the gene level. Further study will be needed to clarify at the protein levels, including dominant ligands for chondrogenesis. Finally, the endpoint of in vivo study was 3-6 weeks, which is insufficient to evaluate the actual fate of neo-tissue. Therefore, we could not declare that mSSC formed cartilaginous tissue can stably function as articular cartilage. Moreover, constituent changes in hSSCs forming fibrous or fibrocartilaginous tissues to form hyaline-like cartilage should be pursued further.

\section{Conclusion}

Adequate chondrogenic factors driving chondrogenesis from somatic stem cells are intrinsically distinct among cell types and species. The chondrogenic response to TGF $\beta 1$ potentially represents the in vivo chondrogenic potential after transplantation into osteochondral defects. Our findings may provide an indicator to predict cell reparability for cartilage diseases prior to clinical use.

\section{Abbreviations}

mASCs; mouse adipose stem cells

mSSCs; mouse synovial stem cells

hASCs; human adipose stem cells

hSSCs; human synovial stem cells

PBMCs; peripheral blood mononuclear cells

BMP; bone morphogenic protein

TGF $\beta$; transforming growth factor beta

Saf-0; Safranin-0

AB; Alcian blue

GFP; Green fluorescent protein

COL; collagen 
ACAN; aggrecan

hVim; human vimentin

TRAP; Tartrate-resistant acid phosphatase

SB; SB-431542

LDN; LDN-193189

OA; osteoarthritis

RA; rheumatoid arthritis

TKA; total knee arthroplasty

\section{Declarations}

\section{Ethics approval and consent to participate}

After written informed consent was obtained from all patients, surgical wastes were used in this study. The protocol was approved by the ethics committee of the University of Tokyo (No.0622-12). Animal experiments were also approved by the University of Tokyo (No.P17-091).

\section{Data Availability Statement}

Nucleotide sequence data reported are available in the DDBJ Sequenced Read Archive under the accession number DRA011462. Other data that support the findings of this study are available from the corresponding author upon reasonable request.

\section{Competing interests}

The authors declare that they have no competing interests.

\section{Consent for publication}

Not applicable.

\section{Fundings}

This work was supported by JSPS KAKENHI (Grand Numbers: 20K18053, 18J01264, and 19H05565), The Nakatomi Foundation, and the Takeda Science Foundation.

\section{Author Contribution:}

Conception: Chijimatsu Ryota, Tanaka Sakae, Saito Taku 
Design of the work: Chijimatsu Ryota, Miwa Satoshi, Okamura Gensuke, Saito Taku

Acquisition and analysis: Chijimatsu Ryota, Miwa Satoshi, Okamura Gensuke, Miyahara Junya, Hisatoshi Ishikura, Higuchi Junya, Maenohara Yuji, Tsuji Shinsaku, Sameshima Shin, Takagi Kentaro, Nakazato Keiu, Kawaguchi Kohei, Yamagami Ryota, Inui Hiroshi, Taketomi Shuji

Interpretation of data: Chijimatsu Ryota, Miwa Satoshi, Okamura Gensuke, Saito Taku

Manuscript writing and revision: Chijimatsu Ryota, Saito Taku

Final approval of manuscript: all of authors.

Agreement to own contributions: all of authors.

\section{Acknowledgment}

We are grateful to Sayaka Ogikubo, Reiko Honma, and Miki Suzuki for their technical support, Jihoon Shin for his technical suggestion, and Zeynep Bal for writing the manuscript. We thank all members of the laboratory for their helpful discussion and comments.

\section{References}

1 Bornes TD, Adesida AB, Jomha NM. Mesenchymal stem cells in the treatment of traumatic articular cartilage defects: a comprehensive review [in eng]. Arthritis Res Ther 2014;16(5):432.

2 Ogura T, Mizuno S, Tsuchiya A. Ongoing studies of cell-based therapies for articular cartilage defects in Japan. Orthop Res Rev 2014:1.

3 Koga H, Muneta T, Nagase T et al. Comparison of mesenchymal tissues-derived stem cells for in vivo chondrogenesis: suitable conditions for cell therapy of cartilage defects in rabbit. Cell Tissue Res 2008;333(2):207-215.

$4 \mathrm{Koga} \mathrm{H}$, Muneta $\mathrm{T}$, Ju YJ et al. Synovial stem cells are regionally specified according to local microenvironments after implantation for cartilage regeneration. Stem Cells 2007;25(3):689-696.

5 Nakamura T, Sekiya I, Muneta T et al. Arthroscopic, histological and MRI analyses of cartilage repair after a minimally invasive method of transplantation of allogeneic synovial mesenchymal stromal cells into cartilage defects in pigs. Cytotherapy 2012;14(3):327-338.

6 Fellows CR, Matta C, Zakany R et al. Adipose, Bone Marrow and Synovial Joint-Derived Mesenchymal Stem Cells for Cartilage Repair [in eng]. Front Genet 2016;7:213.

7 Spees JL, Lee RH, Gregory CA. Mechanisms of mesenchymal stem/stromal cell function [in eng]. Stem Cell Res Ther 2016;7(1):125. 
8 Reinisch A, Etchart N, Thomas D et al. Epigenetic and in vivo comparison of diverse MSC sources reveals an endochondral signature for human hematopoietic niche formation. Blood 2015;125(2):249260.

9 Sakurai H, Sakaguchi Y, Shoji E et al. In vitro modeling of paraxial mesodermal progenitors derived from induced pluripotent stem cells. PLoS One 2012;7(10):e47078.

10 Isobe $\mathrm{Y}$, Koyama N, Nakao $\mathrm{K}$ et al. Comparison of human mesenchymal stem cells derived from bone marrow, synovial fluid, adult dental pulp, and exfoliated deciduous tooth pulp. Int J Oral Maxillofac Surg 2016;45(1):124-131.

11 Sakaguchi Y, Sekiya I, Yagishita K et al. Comparison of human stem cells derived from various mesenchymal tissues: superiority of synovium as a cell source. Arthritis Rheum 2005;52(8):2521-2529.

12 Yoshimura $H$, Muneta $T$, Nimura A et al. Comparison of rat mesenchymal stem cells derived from bone marrow, synovium, periosteum, adipose tissue, and muscle. Cell Tissue Res 2007;327(3):449-462.

13 Futami I, Ishijima M, Kaneko H et al. Isolation and characterization of multipotential mesenchymal cells from the mouse synovium [in eng]. PLoS One 2012;7(9):e45517.

14 Sasaki A, Mizuno M, Ozeki N et al. Canine mesenchymal stem cells from synovium have a higher chondrogenic potential than those from infrapatellar fat pad, adipose tissue, and bone marrow [in eng]. PLoS One 2018;13(8):e0202922.

15 Sekiya I, Muneta T, Horie M et al. Arthroscopic Transplantation of Synovial Stem Cells Improves Clinical Outcomes in Knees With Cartilage Defects [in eng]. Clin Orthop Relat Res 2015;473(7):2316-2326.

16 Shimomura K, Yasui Y, Koizumi K et al. First-in-Human Pilot Study of Implantation of a Scaffold-Free Tissue-Engineered Construct Generated From Autologous Synovial Mesenchymal Stem Cells for Repair of Knee Chondral Lesions. The American Journal of Sports Medicine 2018;46(10):10.

17 Dickhut A, Pelttari K, Janicki P et al. Calcification or dedifferentiation: requirement to lock mesenchymal stem cells in a desired differentiation stage [in eng]. J Cell Physiol 2009;219(1):219-226.

18 Chijimatsu R, Yano F, Saito T et al. Effect of the small compound TD-198946 on glycosaminoglycan synthesis and transforming growth factor beta3-associated chondrogenesis of human synovium-derived stem cells in vitro [in eng]. J Tissue Eng Regen Med 2019.

19 Chijimatsu R, Kobayashi M, Ebina K et al. Impact of dexamethasone concentration on cartilage tissue formation from human synovial derived stem cells in vitro [journal article]. Cytotechnology 2018;70(2).

20 Shintani N, Hunziker EB. Differential effects of dexamethasone on the chondrogenesis of mesenchymal stromal cells: influence of microenvironment, tissue origin and growth factor [in eng]. Eur Cell Mater 2011;22:302-319; discussion 319-320. 
21 Shirasawa S, Sekiya I, Sakaguchi Y et al. In vitro chondrogenesis of human synovium-derived mesenchymal stem cells: optimal condition and comparison with bone marrow-derived cells [in eng]. J Cell Biochem 2006;97(1):84-97.

22 Chijimatsu R, Ikeya M, Yasui Y et al. Characterization of Mesenchymal Stem Cell-Like Cells Derived From Human iPSCs via Neural Crest Development and Their Application for Osteochondral Repair. Stem Cells Int 2017;2017:1960965.

23 Liu Y, Buckley CT, Almeida HV et al. Infrapatellar fat pad-derived stem cells maintain their chondrogenic capacity in disease and can be used to engineer cartilaginous grafts of clinically relevant dimensions [in eng]. Tissue engineering Part A 2014;20(21-22):3050-3062.

24 Tangtrongsup S, Kisiday JD. Modulating the oxidative environment during mesenchymal stem cells chondrogenesis with serum increases collagen accumulation in agarose culture [in eng]. $J$ Orthop Res 2018;36(1):506-514.

25 Lee S, Kim JH, Jo CH et al. Effect of serum and growth factors on chondrogenic differentiation of synovium-derived stromal cells [in Eng]. Tissue engineering Part A 2009;15(11):3401-3415.

26 Moriguchi Y, Tateishi K, Ando W et al. Repair of meniscal lesions using a scaffold-free tissueengineered construct derived from allogenic synovial MSCs in a miniature swine model. Biomaterials 2013;34(9):2185-2193.

27 Sugimoto H, Murahashi Y, Chijimatsu R et al. Primary culture of mouse adipose and fibrous synovial fibroblasts under normoxic and hypoxic conditions. Biomedical Research 2020;41(1):43-51.

28 Caroti CM, Ahn H, Salazar HF et al. A Novel Technique for Accelerated Culture of Murine Mesenchymal Stem Cells that Allows for Sustained Multipotency. Sci Rep 2017;7(1):13334.

29 Murahashi Y, Yano F, Nakamoto H et al. Multi-layered PLLA-nanosheets loaded with FGF-2 induce robust bone regeneration with controlled release in critical-sized mouse femoral defects. Acta Biomater 2019;85:172-179.

30 Saito T, Fukai A, Mabuchi A et al. Transcriptional regulation of endochondral ossification by HIF2alpha during skeletal growth and osteoarthritis development. Nat Med 2010;16(6):678-686.

31 Martin I, Jakob M, Schafer D et al. Quantitative analysis of gene expression in human articular cartilage from normal and osteoarthritic joints [in eng]. Osteoarthritis Cartilage 2001;9(2):112-118.

32 Hildner F, Peterbauer A, Wolbank S et al. FGF-2 abolishes the chondrogenic effect of combined BMP-6 and TGF-beta in human adipose derived stem cells [in eng]. J Biomed Mater Res A 2010;94(3):978-987.

33 Shintani N, Siebenrock KA, Hunziker EB. TGF-ß1 Enhances the BMP-2-Induced Chondrogenesis of Bovine Synovial Explants and Arrests Downstream Differentiation at an Early Stage of Hypertrophy. PLoS 
One 2013;8(1):e53086.

34 Derynck R, Budi EH. Specificity, versatility, and control of TGF- $\beta$ family signaling. Science Signaling 2019;12(570):eaav5183.

35 Kondo S, Muneta T, Nakagawa Y et al. Transplantation of autologous synovial mesenchymal stem cells promotes meniscus regeneration in aged primates [in eng]. J Orthop Res 2017;35(6):1274-1282.

36 Nakagawa Y, Muneta T, Kondo S et al. Synovial mesenchymal stem cells promote healing after meniscal repair in microminipigs [in eng]. Osteoarthritis Cartilage 2015;23(6):1007-1017.

37 Grad S, Loparic M, Peter R et al. Sliding motion modulates stiffness and friction coefficient at the surface of tissue engineered cartilage. Osteoarthritis Cartilage 2012;20(4):288-295.

38 Nakajima M, Wakitani S, Harada Y et al. In vivo mechanical condition plays an important role for appearance of cartilage tissue in ES cell transplanted joint. J Orthop Res 2008;26(1):10-17.

39 Sivasubramaniyan K, Koevoet W, Hakimiyan AA et al. Cell-surface markers identify tissue resident multipotential stem/stromal cell subsets in synovial intimal and sub-intimal compartments with distinct chondrogenic properties [in eng]. Osteoarthritis Cartilage 2019;27(12):1831-1840.

40 Dexheimer V, Gabler J, Bomans K et al. Differential expression of TGF-beta superfamily members and role of Smad1/5/9-signalling in chondral versus endochondral chondrocyte differentiation. Sci Rep 2016;6:36655.

41 Holtzhausen A, Golzio C, How T et al. Novel bone morphogenetic protein signaling through Smad2 and Smad3 to regulate cancer progression and development [in eng]. FASEB journal : official publication of the Federation of American Societies for Experimental Biology 2014;28(3):1248-1267.

42 Miller DSJ, Schmierer B, Hill CS. TGF- $\beta$ family ligands exhibit distinct signalling dynamics that are driven by receptor localisation. J Cell Sci 2019;132(14):jcs234039.

43 Blaney Davidson EN, Vitters EL, van der Kraan PM et al. Expression of transforming growth factor-beta (TGFbeta) and the TGFbeta signalling molecule SMAD-2P in spontaneous and instability-induced osteoarthritis: role in cartilage degradation, chondrogenesis and osteophyte formation [in eng]. Ann Rheum Dis 2006;65(11):1414-1421.

44 Blaney Davidson EN, Remst DF, Vitters EL et al. Increase in ALK1/ALK5 ratio as a cause for elevated MMP-13 expression in osteoarthritis in humans and mice [in eng]. J Immunol 2009;182(12):7937-7945.

45 van der Kraan PM, Goumans MJ, Blaney Davidson E et al. Age-dependent alteration of TGF-beta signalling in osteoarthritis [in eng]. Cell Tissue Res 2012;347(1):257-265.

46 Bush JR, Beier F. TGF- $\beta$ and osteoarthritis-the good and the bad. Nat Med 2013;19:667. 
47 Sundqvist A, Morikawa M, Ren J et al. JUNB governs a feed-forward network of TGF $\beta$ signaling that aggravates breast cancer invasion [in eng]. Nucleic Acids Res 2018;46(3):1180-1195.

48 Coricor G, Serra R. TGF- $\beta$ regulates phosphorylation and stabilization of Sox9 protein in chondrocytes through p38 and Smad dependent mechanisms. Sci Rep 2016;6(1):38616.

49 Baffi MO, Slattery E, Sohn P et al. Conditional deletion of the TGF-beta type II receptor in Col2a expressing cells results in defects in the axial skeleton without alterations in chondrocyte differentiation or embryonic development of long bones [in eng]. Dev Biol 2004;276(1):124-142.

50 Spagnoli A, O'Rear L, Chandler RL et al. TGF-beta signaling is essential for joint morphogenesis [in eng]. J Cell Biol 2007;177(6):1105-1117.

51 Shen J, Li J, Wang B et al. Deletion of the transforming growth factor beta receptor type II gene in articular chondrocytes leads to a progressive osteoarthritis-like phenotype in mice [in eng]. Arthritis Rheum 2013;65(12):3107-3119.

52 Maenohara Y, Chijimatsu R, Tachibana N et al. Lubricin Contributes to Homeostasis of Articular Cartilage by Modulating Differentiation of Superficial Zone Cells. J Bone Miner Res 2020;n/a(n/a).

53 Albro MB, Cigan AD, Nims RJ et al. Shearing of synovial fluid activates latent TGF- $\beta$ [in eng]. Osteoarthritis Cartilage 2012;20(11):1374-1382.

54 Albro Michael B, Nims Robert J, Cigan Alexander D et al. Accumulation of Exogenous Activated TGF- $\beta$ in the Superficial Zone of Articular Cartilage. Biophys J 2013;104(8):1794-1804.

55 Barry F, Boynton RE, Liu B et al. Chondrogenic differentiation of mesenchymal stem cells from bone marrow: differentiation-dependent gene expression of matrix components [in eng]. Exp Cell Res 2001;268(2):189-200.

56 Huey DJ, Hu JC, Athanasiou KA. Unlike bone, cartilage regeneration remains elusive [in eng]. Science 2012;338(6109):917-921.

57 Engels PE, Tremp M, Kingham PJ et al. Harvest site influences the growth properties of adipose derived stem cells [journal article]. Cytotechnology 2013;65(3):437-445.

58 Rezai Rad M, Bohloli M, Akhavan Rahnama M et al. Impact of Tissue Harvesting Sites on the Cellular Behaviors of Adipose-Derived Stem Cells: Implication for Bone Tissue Engineering. Stem Cells Int 2017;2017:2156478.

$59 \mathrm{Ng}$ J, Little CB, Woods S et al. Stem cell directed therapies for osteoarthritis: The promise and the practice: Concise review. Stem Cells 2019;n/a(n/a). 
60 Petrenko Y, Vackova I, Kekulova K et al. A Comparative Analysis of Multipotent Mesenchymal Stromal Cells derived from Different Sources, with a Focus on Neuroregenerative Potential. Sci Rep 2020;10(1):4290.

61 O'Connell JX. Pathology of the synovium [in eng]. Am J Clin Pathol 2000;114(5):773-784.

62 Riis RGC, Gudbergsen H, Simonsen $\mathrm{O}$ et al. The association between histological, macroscopic and magnetic resonance imaging assessed synovitis in end-stage knee osteoarthritis: a cross-sectional study. Osteoarthritis Cartilage 2017;25(2):272-280.

63 Mochizuki T, Muneta T, Sakaguchi Y et al. Higher chondrogenic potential of fibrous synovium- and adipose synovium-derived cells compared with subcutaneous fat-derived cells: distinguishing properties of mesenchymal stem cells in humans. Arthritis Rheum 2006;54(3):843-853.

64 Mizuno M, Katano H, Mabuchi Y et al. Specific markers and properties of synovial mesenchymal stem cells in the surface, stromal, and perivascular regions [in eng]. Stem Cell Res Ther 2018;9(1):123.

65 Neumann E, Lefèvre S, Zimmermann B et al. Rheumatoid arthritis progression mediated by activated synovial fibroblasts. Trends Mol Med 2010;16(10):458-468.

66 Chou C-H, Jain V, Gibson J et al. Synovial cell cross-talk with cartilage plays a major role in the pathogenesis of osteoarthritis. Sci Rep 2020;10(1):10868.

67 Tsuchiya H, Ota M, Sumitomo S et al. Parsing multiomics landscape of activated synovial fibroblasts highlights drug targets linked to genetic risk of rheumatoid arthritis. Ann Rheum Dis 2020:annrheumdis2020-218189.

68 Dicarlo M, Teti G, Cerqueni G et al. Synovium-derived stromal cell-induced osteoclastogenesis: a potential osteoarthritis trigger [in eng]. Clin Sci (Lond) 2019;133(16):1813-1824.

69 Okamura G, Ebina K, Hirao M et al. Promoting Effect of Basic Fibroblast Growth Factor in Synovial Mesenchymal Stem Cell-Based Cartilage Regeneration. Int J Mol Sci 2021;22(1):300.

70 Caplan Al. Mesenchymal Stem Cells: Time to Change the Name! [in eng]. Stem cells translational medicine 2017;6(6):1445-1451.

\section{Supplementary Files}

This is a list of supplementary files associated with this preprint. Click to download.

- Additionalfile1.xlsx

- Additionalfile2.xlsx

- Additionalfile3.xlsx 
- Additionalfile4.pptx

- Additionalfile5.pptx

- Additionalfile6.pptx

- Additionalfile7.pptx

- Additionalfile8.pptx

- Additionalfile9.pptx

- Additionalfile10.pptx

- Additionalfile11.pptx

- Additionalfile12.pptx

- Additionalfile13.pptx

- Additionalfile14.pptx

- Additionalfile15.pptx

- Additionalfile16.pptx

- Additionalfile17.xlsx

- Additionalfile18.pptx

- Additionalfile19.pptx

- Additionalfile20.pptx

- Additionalfile21.pptx

- Supplementarylegends.docx 